\title{
Thunderstorm nowcasting by means of lightning and radar data: algorithms and applications in northern Italy
}

\author{
P. Bonelli and P. Marcacci \\ CESI RICERCA, Milano, Italy
}

Received: 27 February 2008 - Revised: 14 July 2008 - Accepted: 9 September 2008 - Published: 28 October 2008

\begin{abstract}
Thunderstorms and their ground effects, such as flash floods, hail, lightning, strong winds, and tornadoes, are responsible for most weather damages in northern Italy, especially in the warm season from May to September. A nowcasting and warning system focused on severe thunderstorm events would be useful to reduce risks for people involved in outside activities and for electric, telecommunication, and sensitive industrial business. C-band radar and Lighting Location Systems provide useful, fast and high resolution data for the detection of convective systems and for following their dynamics. The whole of northern Italy is covered by radar with a resolution of $1 \mathrm{~km}$ and by a lightning network with a mean accuracy of $0.5 \mathrm{~km}$ on the single point of impact. The authors present an algorithm developed for tracking high intensity storm cells by means of radar and lightning data. Application to northern Italy reveals that tracking thunderstorm cells can be used as an alert system that may help prevent damages from extreme weather, as well as allowing for studying the correlation among lightning, rainfall and tornado occurrence. Assessing the algorithm skill is also discussed, and a forecast verification method is described and applied for the duration of a thunderstorm season.
\end{abstract}

\section{Introduction}

Thunderstorms and their ground effects, such as flash floods, hail, lightning, strong wind, and tornadoes, are responsible for most weather damages in northern Italy, especially in the warm season from May to September. On the other hand, in the rest of Italy, they occur mostly in the cold season.

The immediate area affected by a single thunderstorm, in the short time of its life cycle, may be only a few kilometers,

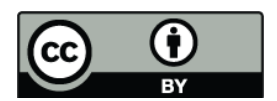

Correspondence to: P. Bonelli

(pbonelli@ cesiricerca.it) and its related phenomena at the ground may last for only an hour locally (though the whole convective system can move horizontally for several tens of kilometers and be dangerous for some hours). Despite these relatively small space-time dimensions, however, thunderstorms have a strong impact on the densely populated regions on the northern Italian plain, and, even more strongly, on the boundary region between the plain and the Alpine Chain, where the frequency and the intensity of these events are very high due to the forced slope lifting.

Following these considerations, local authorities and electrical utilities are interested in a system able to forecast and give warning when severe thunderstorm events are to occur, to reduce risks for people involved in outdoor activities, and for electric, telecommunication, and sensitive industrial business. In addition, a documentation system that collects information about when and where thunderstorms have occurred is very important for people seeking compensation for suffered damages when they need to legally demonstrate the cause of these damages.

In general, forecasting thunderstorms for very short time periods is possible using high resolution observation systems that give a detailed description of some physical variables related to convective clouds. For this reason the term "nowcasting" is often used. A status report of thunderstorms nowcasting is provided by Wilson (Wilson et al., 1998; Wilson, 2004).

The great variety of nowcasting operational radar systems found in the scientific literature reflects the differences in observational facilities and meteorological organizations available for the application area. Furthermore, the specific needs of the final users can force the choice of the method to be implemented.

Some automatic nowcasting techniques recognize high reflectivity radar patterns, track them in time, and extrapolate them for the next short time period (e.g. half an hour), following a steady state assumption. Other methods introduce

Published by Copernicus Publications on behalf of the European Geosciences Union. 
an evolution extrapolation as well. The tracking techniques can be different too: cross correlation is used generally to compute wide precipitation pattern advection (Poli et al., 2005), instead of mass centroid methods that are more accurate for following small strong cells (Johnson et al., 1998). A third approach, recently proposed by $\mathrm{Xu}$ and Chandrasekhar (2005), derives the whole motion field from spectral analysis of the radar field.

Generally, to detect, evaluate, track and forecast thunderstorms we need a very high resolution observation system, and automatic algorithms applied to these data, to obtain quantitative information on the position, size, path and velocity of the cell. In northern Italy, the first need is satisfied by the availability of some C-band radar operated by different regional met-services, and one radar operated by Meteo Swiss, that cover essentially all the territory, although they are not yet interconnected. Furthermore the Italian Lightning Location System (LLS), named SIRF and operated by CESI, is a very high resolution system for detecting cloud to ground lightning in real time (Bernardi et al., 2004).

The second need, for algorithms, is the object of this paper. This need can be satisfied by computer procedures that perform the following tasks:

- Recognizing highest reflectivity areas (thunderstorm cells) or highest lightning density areas;

- Tracking and extrapolating cell motion;

- Issuing alarm messages toward different users;

- Computing scores, from a comparison among observed and forecasted cell positions, after a period of operation.

The choice of tracking individual small cells is justified by the complexity of the mountainous area of interest (Hering et al., 2005) and the lack of a coordinated radar network in Italy able to detect larger systems.

At CESI RICERCA we started to approach the problem by tracking small, strong thunderstorms and forecasting their motion for a short time, half an hour, without taking into account the growth/decay of a cell. We used the rainfall field estimate $(\mathrm{mm} / \mathrm{h})$ from the Mount Lema Meteo Swiss C-band radar (Joss et al., 1997), covering a large area on the south of Alps, because of the consideration that the highest rainfall rate areas (thunderstorm cell) are responsible also for the highest risk of damage due to strong wind, as downburst or tornado. The time step of the rain field was $15 \mathrm{~min}$. Our study area was Lombardia and a part of the Piemonte region.

Considering the availability of lightning Cloud to Ground (CG) data over the whole of Italy and the trans-boundary countries provided by the CESI-SIRF, we developed an algorithm able to independently track radar and lightning data, taking into account that in the presence of thunderstorms, high reflectivity areas are usually the highest density lightning areas. In this work we compared the trajectories computed by both systems. We also developed a real-time pro- cedure able to extrapolate the thunderstorm movement (nowcasting) for half an hour and to issue alert messages related to defined target areas.

Furthermore, a method to assess the algorithm forecast skill is needed in order to tune and improve the algorithm itself. In the paper we describe such a method and how it was tested over the duration of a thunderstorm season.

Finally, we present a case in which the combined use of radar and lightning data has been applied to study the connection between positive polarity lightning and tornado formation.

\section{Cell detection}

The main features of the data used in the present work are:

- rainfall rate $(\mathrm{mm} / \mathrm{h})$ from the M. Lema radar on a regular $1 \mathrm{~km}$ mesh over a domain of $400 \times 400 \mathrm{~km}^{2}$ and a time step of $15 \mathrm{~min}$;

- CG lightning impact coordinates and time and current polarity over the whole radar domain; the position of each lightning strike is assumed to be correct within $0.5 \mathrm{~km}$ and the time with a precision of less than $1 \mathrm{~s}$.

The radar domain contains a large part of northern Italy, see Fig. 1.

In order to apply the tracking algorithm to both radar and lightning data, a pre-processing procedure transforms the irregularly spaced lightning data set into a regular lat/long mesh $0.05^{\circ}$ wide, where each grid box is associated with the number of lightning observations fallen inside it for a period of $15 \mathrm{~min}$. This procedure allows one to treat the lightning observations in the same way as the radar observations, simply taking into account different mesh dimensions and coordinate systems. In the following we will refer to the value of radar or lightning data associated with each grid point as a "pixel." We chose a mesh of about $5 \mathrm{~km}$ for the lightning data, larger than for the radar, because the number of lightning strikes falling in each radar grid box is very small.

A thunderstorm cell is located, on the radar or lightning grid by means of a procedure starting from the maximum pixel value in the whole grid and trying to find a continuous pixel structure around it. When the next pixel value found in the structure falls below a fixed threshold, then the cell is defined and all its attributes can be computed, as described later. Another cell can be detected in the same way, deleting previously all the pixels of the first cell from the grid. The procedure stops when no structure of more then 4 pixels remains in the grid. A fixed threshold of $20 \mathrm{~mm} / \mathrm{h}$ was set for the application described in this paper. This threshold, corresponding to about $44 \mathrm{dBz}$, assures the location of only strong cells, and of attributing to them small dimensions. A fixed threshold is also used in the TITAN algorithm (Dixon et al., 1993); other algorithms, like TRT (Hering, 2004), use a variable adaptive threshold instead. We chose the fixed threshold 


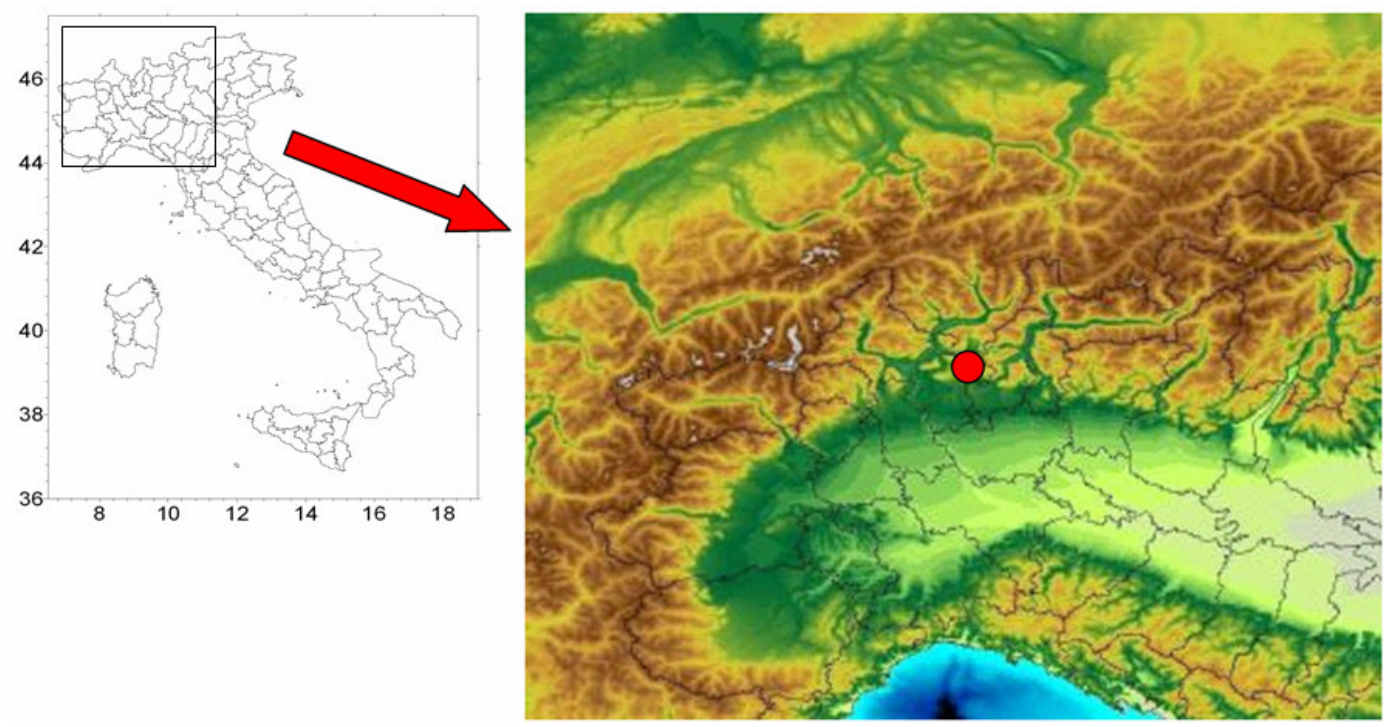

Fig. 1. Study area: $400 \times 400 \mathrm{~km}$ covered by the Monte Lema radar (red dot).

approach as a starting point, with future improvements to be decided on the basis of the application results.

Cell centre is computed by averaging the pixel coordinates weighted by the pixel value (centre of mass). Cell radius is defined as the maximum distance between the centre and the cell pixels. In such a way, thunderstorm cells may have any radius. As a consequence, the cell, so defined, may contain more than one relative maximum that may be independent cells. This is a limitation due to the fixed threshold method that could be removed in a later version of the algorithm.

\section{Tracking}

Cells detected as described in the previous paragraph must be linked between two successive radar frames in order to build a story and a trajectory for each cell. The problem is complicated by the possibilities of a cell being born and dying, moving on a different path from the others, and merging with other cells. This can be handled in various ways. For example, in the TITAN algorithm, the problem of optimization was resolved in order to find an optimal set of paths among all the possible combinations of cells. The TRT algorithm uses overlapping area between successive cells to choose the true couples. Our algorithm, every time step, computes a set of variables for every couple of cells and then attempts to choose the best set of values among all the possibilities.

The most important variable computed between two cells in different radar frames is the displacement of the cell centre of gravity, i.e. the module and direction of the centre velocity.

The velocity module for every couple of cells is:

$V_{i, j}=\frac{\sqrt{\left(x_{i}-x_{j}\right)^{2}+\left(y_{i}-y_{j}\right)^{2}}}{\Delta t}$ $\left(i=1, . . N_{1} ; j=1, . . N_{2}\right)$

Where $\left(x_{i} ; y_{i}\right)$ are the coordinate of the cell " $i$ " centre at time $(t+\Delta t)$ and $\left(x_{j} ; y_{j}\right)$ those of the cell " $j$ " at time $(t)$. $N_{1} ; N_{2}$ are the numbers of the cells identified respectively in the $(t+\Delta t)$ and $(t)$ frame.

The direction angle:

$D_{i, j}=\tan ^{-1} \frac{\left(y_{i}-y_{j}\right)}{\left(x_{i}-x_{j}\right)}\left(0^{\circ}<D_{i, j} \leq 360^{\circ}\right)$

$\left(i=1, . . N_{1} ; j=1, . . N_{2}\right) ;\left(V_{i, j} \geq V_{\min }\right)$

Where $V_{\min }$ represents a minimum velocity value for which direction angle has a sense: it is set to $2 \mathrm{~m} / \mathrm{s}$.

Other variables complete the set computed for every couple:

The absolute value of the difference between the velocity modules:

$\Delta V_{i, j}=\left|V_{i, j}-V_{j}\right|$;

the absolute value of the difference between the direction angles:

$\Delta D_{i, j}=\left|D_{i, j}-D_{j}\right| \quad 0^{\circ} \leq \Delta D_{i, j} \leq 180^{\circ} ;$

where $V_{j}$ and $D_{j}$ are the velocity and direction of cell " $j$ " computed in the previous time step.

The shape similarity:

$C_{i, j}=c c\left(\{P\}_{i},\{P\}_{j}\right) \quad 0 \leq C_{i, j} \leq 1 ;$

where $\{P\}_{i}$ and $\{P\}_{j}$ are respectively the series of pixel value of the cells $i$ and $j$ (i.e. the rain intensity or the number of lightning strikes). The two series have the same number of pixels and are extracted by a box containing the two cells (see 


\section{Cell "i"}

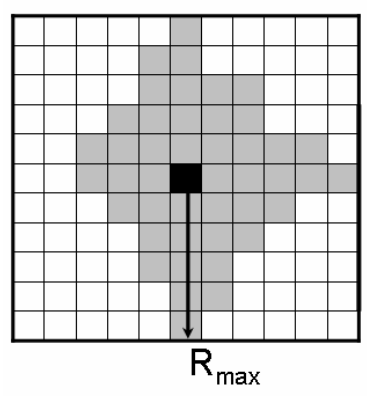

$$
\{P\}_{i} \equiv P_{i, 1}, P_{i, 2}, \ldots P_{i, n}
$$

Cell "j"

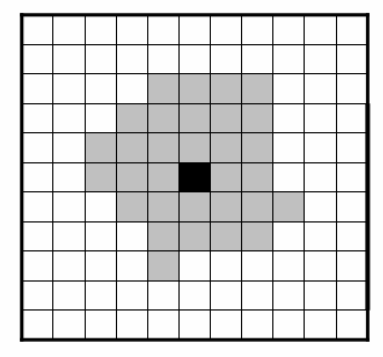

$\{P\}_{j} \equiv P_{j, 1}, P_{j, 2}, \ldots P_{j, n}$
Fig. 2. Computation of the shape similarity between two cells: A box with a dimension equal to the maximum between the two cells' diameters is defined around the centre of each cell. The pixel values inside each box contribute to the computation of the correlation coefficient or the threat score.

Fig. 2). " $c c$ " indicates the correlation coefficient computed among the correspondent pixels of the two cells.

Alternatively to the correlation coefficient, the shape similarity can be computed as a threat score applied to a binary pixel matrix ("0" below a fixed threshold, " 1 " above). In the case study presented in this paper, the threat score method with a threshold of $20 \mathrm{~mm} / \mathrm{h}$ has been used.

Also, the change in the size variable has been computed:

$\Delta n_{i, j}=\frac{\left|n_{i}-n_{j}\right|}{n_{j}}$

where $n_{i} ; n_{j}$ are the numbers of non- 0 pixels in the cells $i$ and $\mathrm{j}$ computed from the two series in Eq. (5).

For each quantity defined in Eqs. (3), (4), (5), and (6), a score from 0 to 100 has been computed by means of score functions. These functions, as shown in Fig. 3, are strongly non-linear (S-shaped). The main purpose of defining score functions is to allow for the subsequent combinations of several physical quantities. A score average is computed from the single scores.

The score functions are defined so as to cause the algorithm to choose the most conservative couples as the true ones. A high score means that the couple has a high probability to be the true one. Figure 3 contains examples of the score functions.

The functions for the quantities defined in Eqs. (3), (4) and (6) have a generalized form:

$$
\begin{aligned}
& S(X)=100 \text { for } X \leq 0 \\
& S(X)=200 \frac{\exp \left(-2 A X^{2}\right)}{\exp (A X)+\exp (-A X)} \text { for } X>0
\end{aligned}
$$

where $X=\Delta V-\Delta V_{\max }$ or $X=\Delta D-\Delta D_{\max } \quad$ or $X=\Delta n-\Delta n_{\max }$.
The score function for the quantity defined in Eq. (5) has the following form:

$$
\begin{aligned}
& S(X)=0 \text { for } X \leq 0 \\
& S(X)=100-200 \frac{\exp \left(-2 A X^{2}\right)}{\exp (A X)+\exp (-A X)} \text { for } X>0
\end{aligned}
$$

where $X=C-C_{\min }$.

The function slope and its position can be modified by varying only the parameter $A$ and the reference values: $\Delta V_{\max } ; \Delta D_{\max } ; \Delta n_{\max } ; C_{\min }$ for the physical quantities. These values must be tuned by means of some case studies.

The algorithm works in the following way: first, it removes all the couples with velocities that are too high, which represent remote cells that have a very low probability to be the same cells moving very fast; second, the score average computed from the other variables is taken into account.

In many cases, when few cells are present in the domain, the first step alone is sufficient to identify the couples for the tracking computation. In other cases, when many cells are present, more couples, candidates to be the true ones, often share the same cells. In this situation the algorithm starts to examine the score average for each couple.

The resultant score matrix is analysed in the following way. First, the algorithm attributes a "priority level" to every couple, that is, the number of times the two cells in one couple are present in the other couples too. Second, the algorithm deletes step by step the couples with a higher priority level and lower score.

Looking at Fig. 4, we see an example of a score matrix at the previous time step, cells A to F, and the actual step, cells 1 to 5. After an initial removal of couples with velocities that are too high (remote cells), the situation is that presented in the figure: every coloured box represents a possible couple with its velocity (first value) and its total score (second value). Different colours identify different priority level. For instance, the red couple 4-C shares the cells 4 and $C$ with the couples $2-\mathrm{C}$ and $4-\mathrm{A}$, and it has a priority level equal to 2 . The green couple $2-\mathrm{C}$ shares a cell only with $4-\mathrm{C}$ (priority level =1), while the yellow couple 3-E does not share any cell (priority level $=0$ ).

The algorithm deletes, step by step, couples with the higher priority level, updates, and stops only when all the couples have priority level equal to 0 . In the first removal loop, the algorithm chooses the highest priority level couples (in the case shown in Fig. 4, only the red one) and removes the one with the minimum score (again only the red one in this example). In the second loop, the situation is the one shown in Fig. 5, where two previously green couples became yellow. The 1-F couple is removed because it has the lowest score among the green ones. Finally, in the third loop, the situation is as shown in Fig. 6. The remaining couples, 1-B, 2-C, 3-E, 4-A and 5-D, are chosen because they do not share 

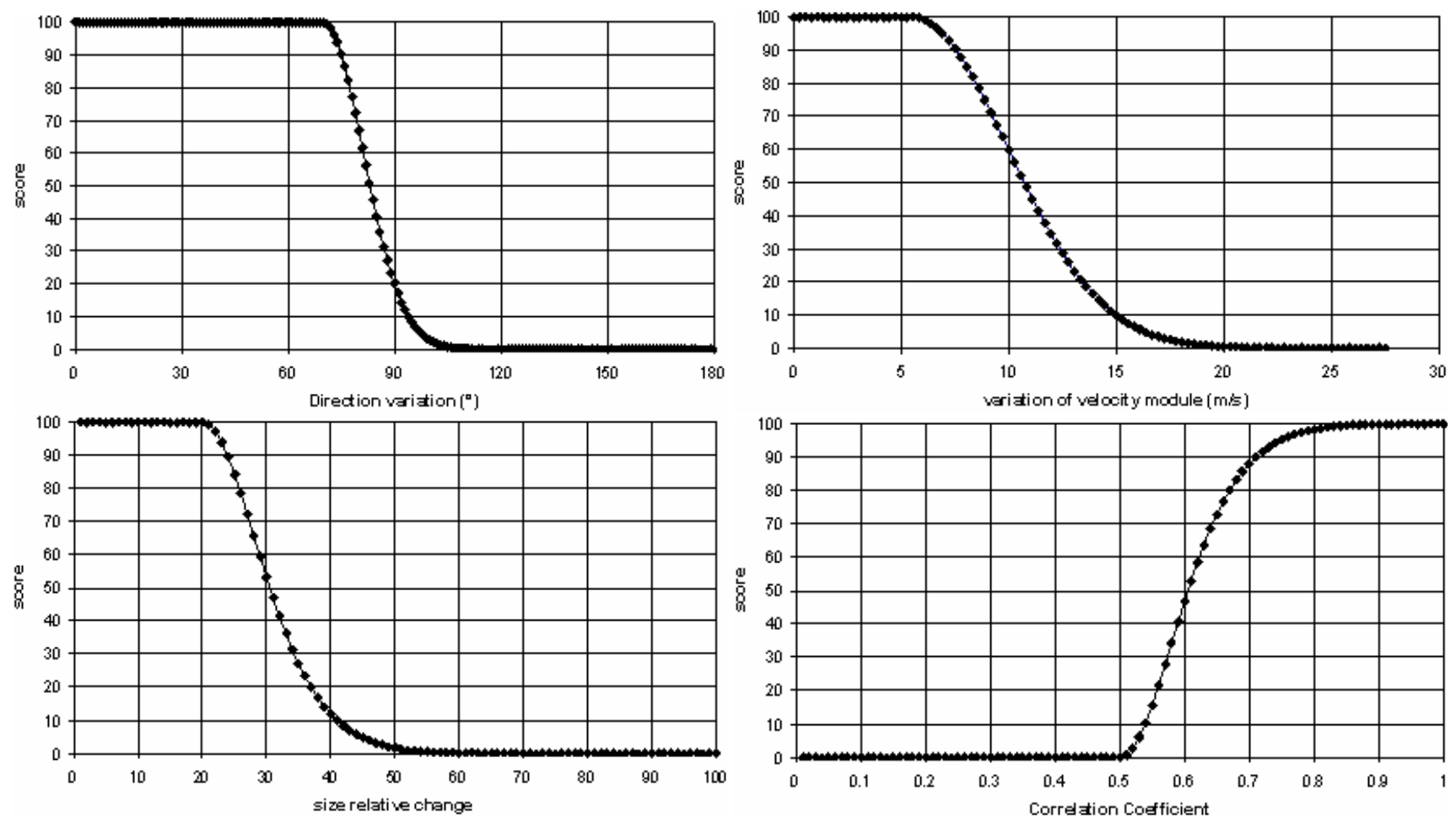

Fig. 3. Functions used to compute a score for every couple of cells on the basis of physical parameters (see text).

any cell with the others. Velocity values for these couples are taken as the cell velocity between the two frames.

This method has the effect of identifying the maximum number of cells that move alone without interacting with each other. If one cell in the previous time step doesn't match any actual cell, it means that the cell has died. By contrast, if an actual cell cannot match any previous cell, it means that it is born and its velocity starts from 0 .

The velocity computed for the remaining couples are used to compute the predicted cell positions after the desired time interval.

\section{Examples for radar and lightning data}

Figure 7 shows a trajectory computed on 13 August 2006 of a single thunderstorm crossing Lombardia from Milano (MI) to Cremona (CR). Figure 8 contains two radar frames representing the tracked cell at different time steps. In this case, the tracking algorithm has no difficulty following the thunderstorm for a long time, without using any score functions.

A more complex case is shown in Fig. 9, for the 29 June 2005, when many thunderstorms, moving in different directions, are caused by a cold front crossing northern Italy. This example shows how the algorithm creates many reasonable trajectories; those in the figure have starting times between 16:00 and 17:00 UTC. Some of them represent single, inde-

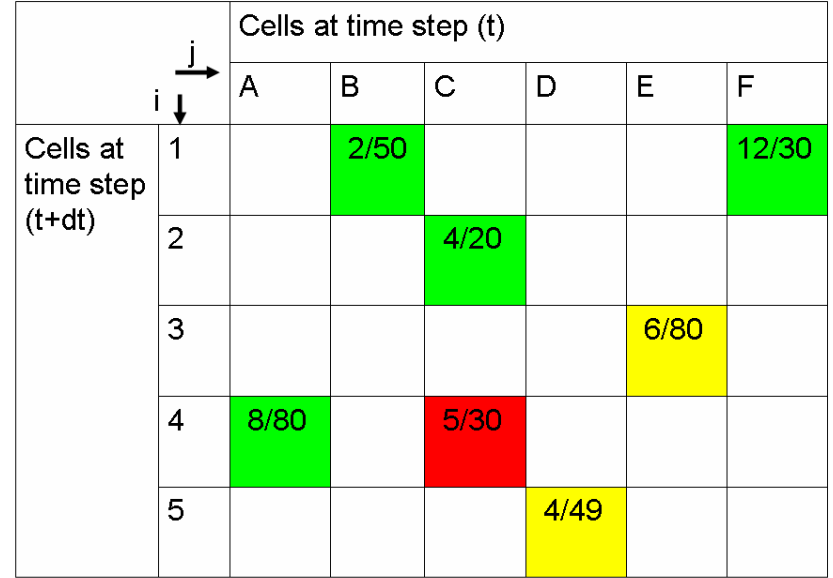

Fig. 4. Example of cell couple matrix at the first step of the selection procedure.

pendent small cells, like those near Biella (BI), and some, having irregular motion, such as the one near Varese (VA), are difficult to track. However, sometimes, even in complex mountainous terrain, as near Trento (TN), a thunderstorm may follow a regular path. Figure 10 shows two radar frames representing the situation described.

The importance of tracking storms by means of lightning data only has been pointed out by some authors, such as 


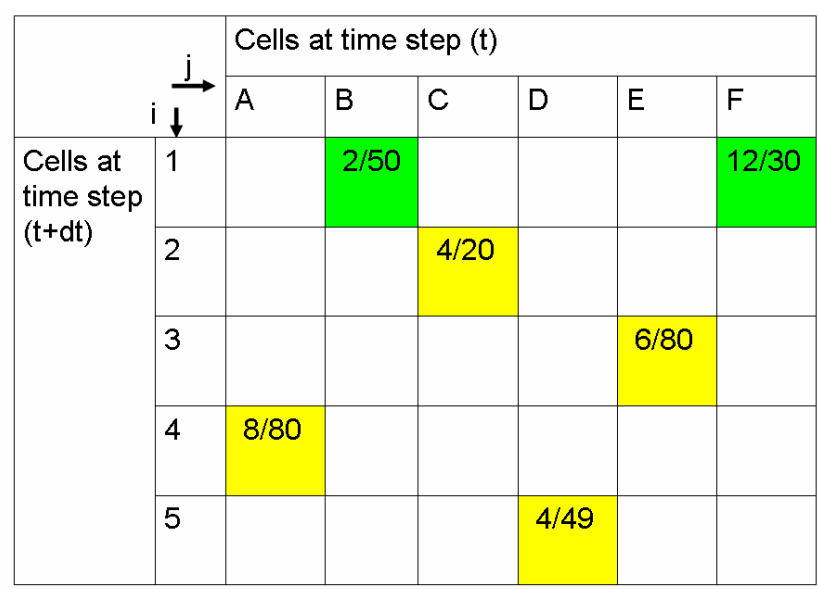

Fig. 5. Example of cell couple matrix at the second step of the selection procedure.

\begin{tabular}{|c|c|c|c|c|c|c|c|}
\hline & & Cells & t time & tep (t) & & & \\
\hline & $i \stackrel{\vec{l}}{\longrightarrow}$ & A & B & c & $D$ & $E$ & 1 \\
\hline Cells at & 1 & & $2 / 50$ & & & & \\
\hline & 2 & & & $4 / 20$ & & & \\
\hline & 3 & & & & & $6 / 80$ & \\
\hline & 4 & $8 / 80$ & & & & & \\
\hline & 5 & & & & $4 / 49$ & & \\
\hline
\end{tabular}

Fig. 6. Example of cell couple matrix at the third step of the selection. procedure.

Bertram (e.g. Bertram et al., 2004), studying thunderstorm climatology over the Alps, or Demetriades (Demetriades et al., 2005), using these data for diagnosing the evolution of convective systems over oceanic areas far from the coast. In the Mediterranean region, lightning data are often the only high resolution data able to monitor convective activity and provide input to tracking algorithms. For this reason, the comparison between radar and lightning trajectories, where radar data are available, is an important task for this research. Therefore, some additional experiments have been carried out using the same tracking algorithm described above.

As stated in the previous paragraph, a pre-processing phase transforms irregularly spaced lightning data, provided by the SIRF system, into a regular grid with a $0.05^{\circ}$ wide mesh and a $15 \mathrm{~min}$ interval. The same trajectory algorithm used for the radar data has been applied to the lightning grid, producing what is shown in Fig. 11, where lightning trajec-

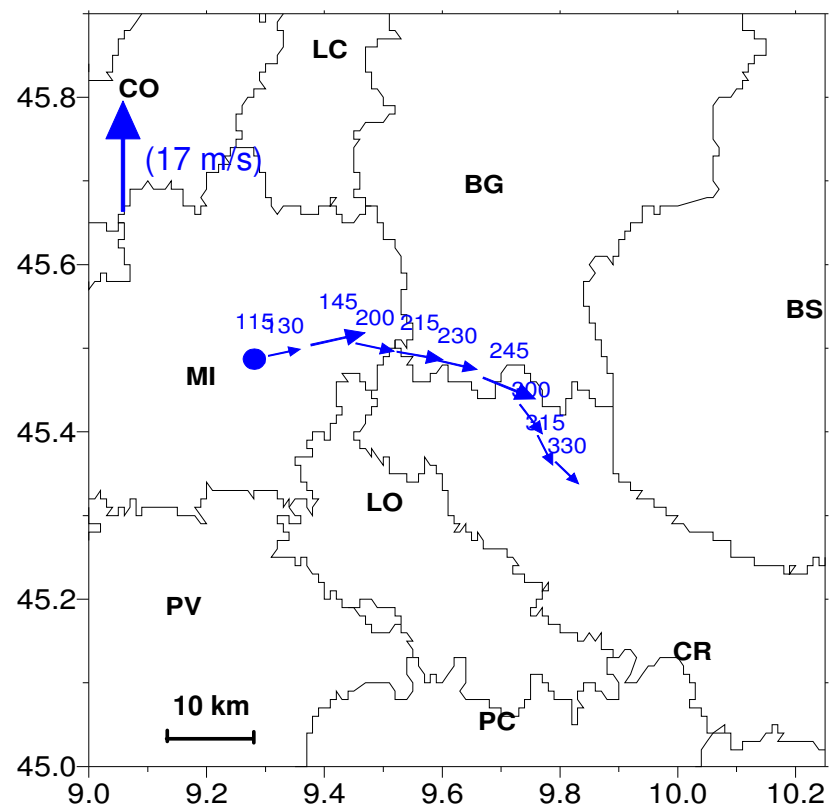

Fig. 7. Thunderstorm trajectory from 01:15 to 03:30 UTC of 13 August 2006 (axis units are in long/lat degree; numbers represent time). The arrow lengths are proportional to the velocity; a reference value is reported on the top left.

tories (red) are represented together with radar ones (blue). The figure shows only trajectories that started since 10:00 to 12:00 UTC.

In some cases, the velocity vectors from lightning and from radar are similar, but we can see that they can also be very different, as at NW and $\mathrm{E}$ of Sondrio (SO) and $\mathrm{W}$ of Bolzano (BZ). In addition, two stationary cells $\mathrm{S}$ of Trento (TN) don't have any lightning.

It is easy to explain large differences in the vector directions when cells are slow, whereas differences both in directions and in velocities may be attributed to some uncertainty in determining the centre of cell with small numbers of lightning strikes or large cells. From these few experiments we can conclude that lightning tracking works better when large mesoscale system are to be tracked.

\section{Lagrangian relations among thunderstorm variables}

One important application of trajectory algorithms is the study of the correlation between rainfall and lightning from a Lagrangian point of view; in other words, the identification of a convective cell during its whole life cycle is an important step in the study of the possible correlations among different variables related to the cell, such as lightning polarity and tornado occurrence. The last topic is extensively treated in the book by MacGorman (MacGorman and Rust, 1998). Some experimental evidence about tornadoes and positive lightning can be found in the work of Tessendorf (Tessendorf et al., 


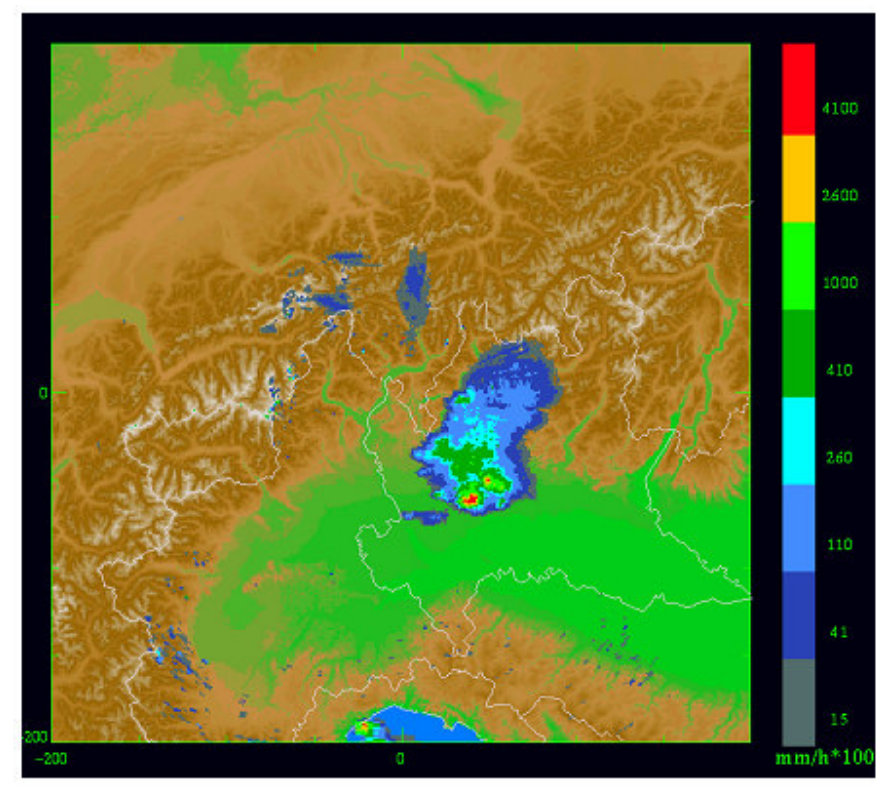

13/08/2006 01:30 UTC

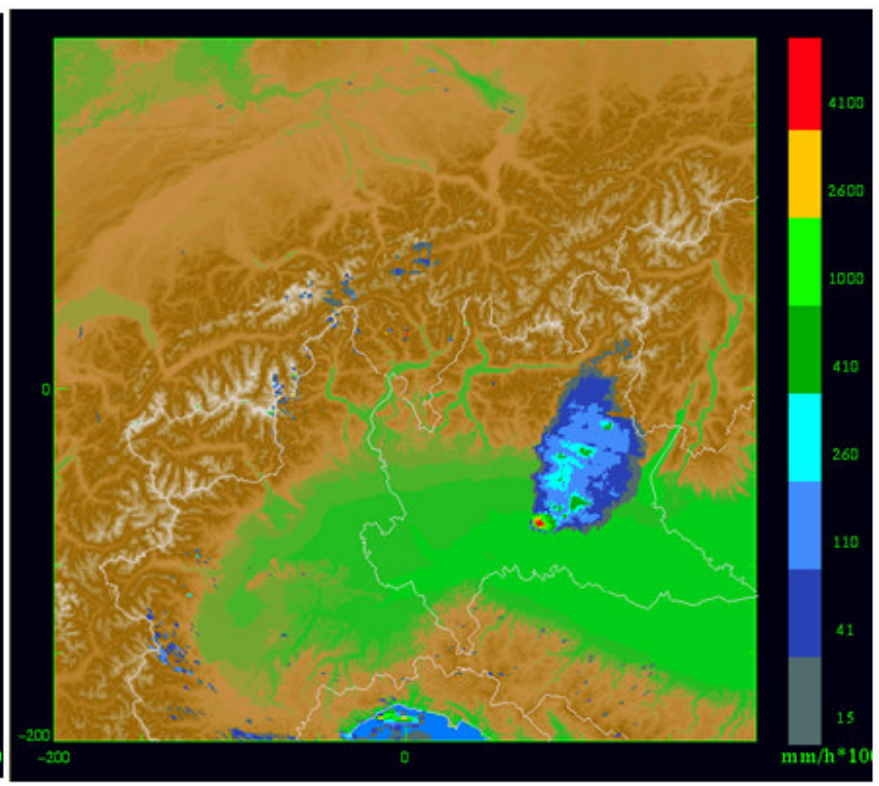

13/08/2006 03:30 UTC

Fig. 8. The 13 August 2006 thunderstorm as shown by two radar frames.

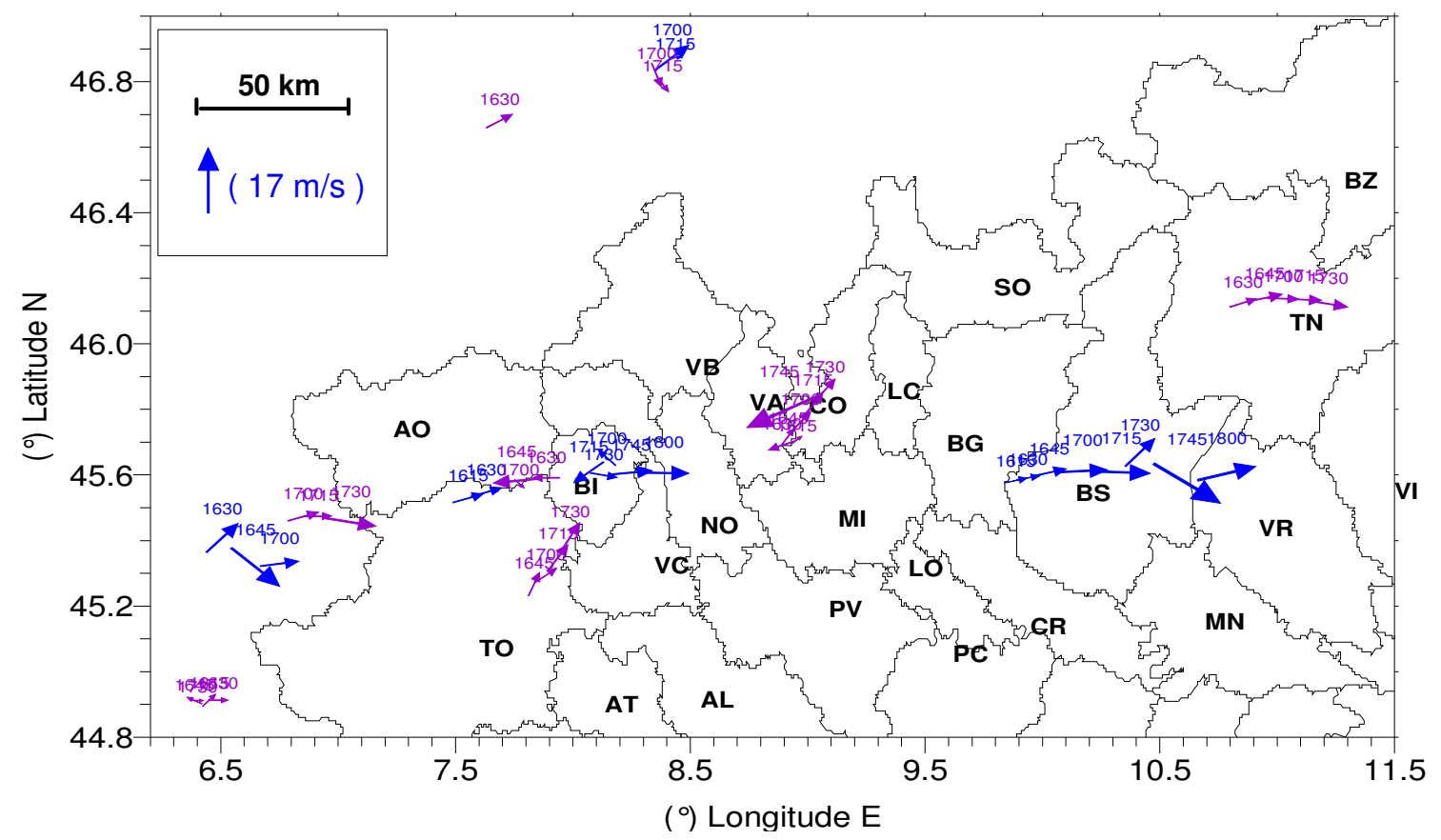

Fig. 9. Many thunderstorm trajectories with starting times between 16:00 and 17:00 UTC on the 29 June 2005; numbers above every arrow refer to time. Different colours distinguish different trajectories located close to each other. The arrow lengths are proportional to the velocity; a reference value is reported on the top left.

2005), who describes a supercell event on 29 June 2000, in Kansas, during the STEPS 2000 campaign; they detected an increase in positive CG flash rate during a tornado touchdown.
A case of a tornado in northern Italy can be seen in Fig. 12, where the number of CG lightning strikes falling inside the cell radius is plotted versus time, separating the positive lightning from the total; rainfall density is plotted as well. 


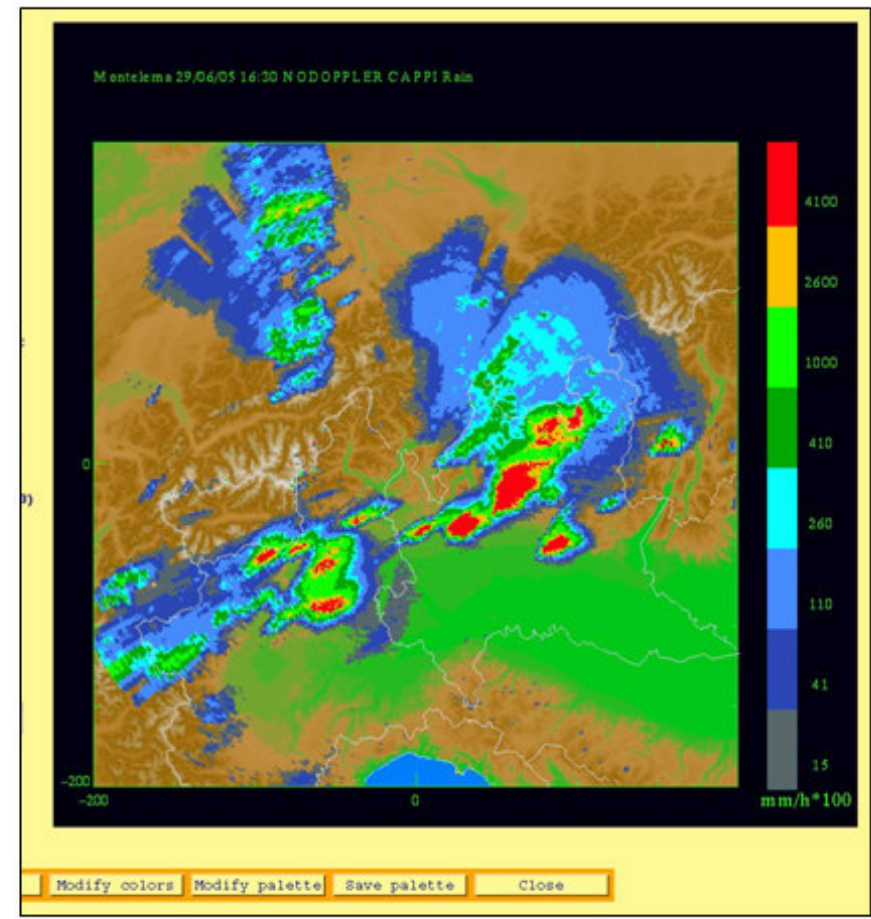

29/06/2005 16:30 UTC

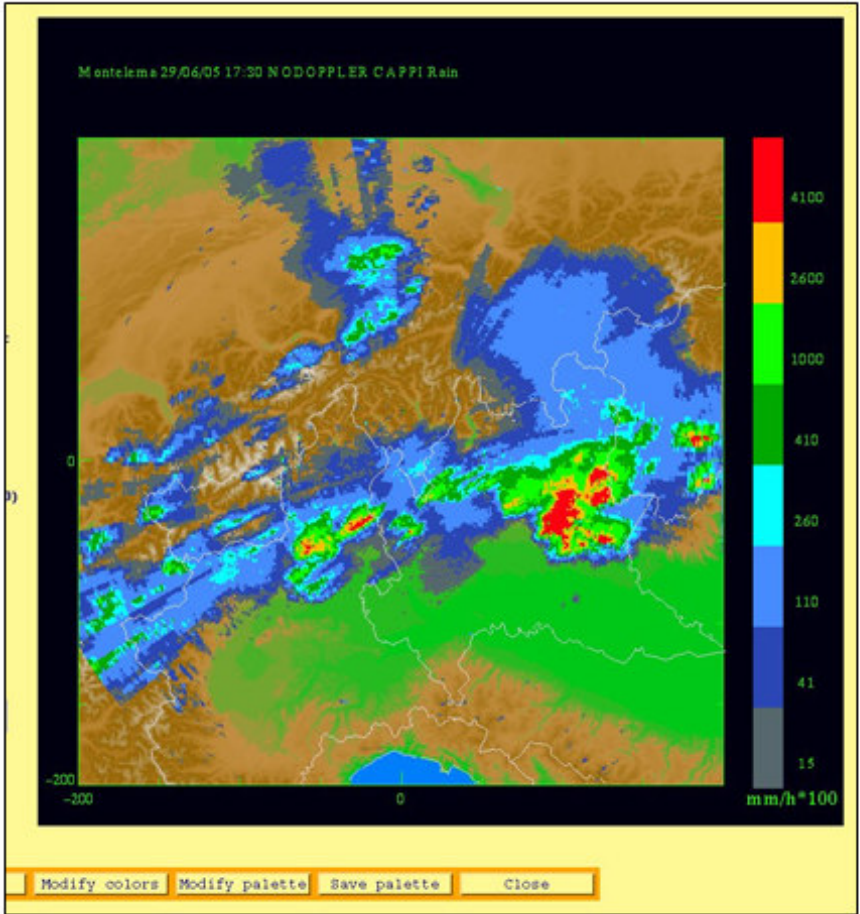

29/06/2005 17:30 UTC

Fig. 10. Two radar frames from the 29 June 2005 showing a complex system of cells crossing the southern side of the Alps.

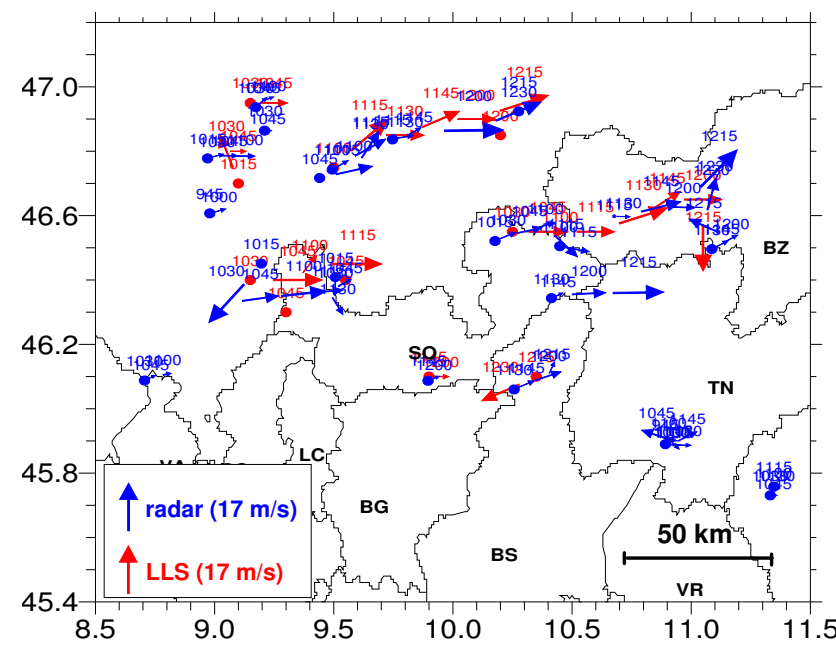

Fig. 11. Case study of 29 June 2005: trajectories starting from 10:00 till 12:00, computed from radar data (blue arrows) and lightning data (red arrows); numbers above the arrows indicate time in UTC. The arrow lengths are proportional to the velocity; a reference value is reported on the bottom left. The circles indicates cells with 0 velocity.
From 18:45 to $19: 15$ UTC of the 29 June 2005, the cell crossed the town of Montorso (near Vicenza) causing a tornado, probably category F1 on the Fujita scale; the roof of the main church in the town was lifted away. In Fig. 12, the label F1 marks the time period in which the tornado is thought to be occurred. The figure shows that the positive lightning density, i.e. the number of lightning strikes divided by the cell area, rises at the beginning of the period, together with a rise in rainfall density. The maximum number of positive CG lightning detected in 15 min was 55, that is, $25 \%$ of the total (negative + positive).

We can state that the amount of positive lightning near Montorso should be considered high and rare, because if we compare the values found in that case with the frequency distribution for all the cells during the whole thunderstorm day, as in Fig. 13, we can see that only $1-2 \%$ of all the cells detected that day have these numbers of positive lightning strikes. This result is in agreement with what is reported by other authors for northern Italy (Bertato et al., 2003) and over the Austrian Alps (Bluestein et al., 1998), allowing us supposing that positive lightning can be a warning signal of high intensity convection events, such as tornadoes. Of course, more cases have to be analyzed before including this factor in an automatic alarm system. 


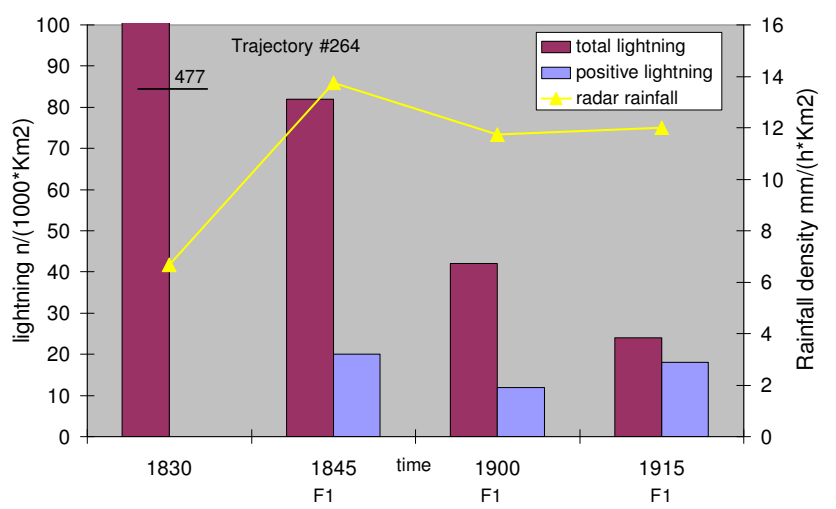

Fig. 12. Time plot with the total (red), positive (blue) lightning density, and rainfall density (yellow) from radar along the cell trajectory for 29 June 2005 near the town of Montorso (Vicenza) where a tornado of probable F1 intensity lifted the roof of a church.

\section{Alert system}

As a test of our algorithm, we thought to include it in an alarm system tailored for real Civil Protection users. These persons usually rescue people attending outdoor public events if various types of adverse situations occur. In order to reduce the minimum time needed to warn these users in case of thunderstorm risk, we carried out an automatic procedure, issuing warning messages via GSM-SMS (mobile telephone short message system) directly to the Civil Protection users. A collaboration with the Regione Lombardia Civil Defence allowed us to test the alert system in the warm season of 2007.

Radar maps were available at CESI RICERCA every $15 \mathrm{~min}$. After few minutes of process time needed to detect cells, to compare them with the earlier ones, and to extrapolate their position using the computed velocity, the system checks if a severe thunderstorm overlaps a pre-defined "target area". If it does, an SMS is issued and transmitted to one or more users expected to be present on the site of the target.

The SMS messages were sent when a thunderstorm cell with a maximum intensity of more than $40 \mathrm{~mm} / \mathrm{h}$ was forecasted to cross or partially overlap a target area within the next $30 \mathrm{~min}$. The targets were identified as circle of $10 \mathrm{~km}$ radius around every main town in Lombardy (12 towns), see Fig. 14. The alarm messages included the position sector of the cell respect to the town $(\mathrm{N}, \mathrm{NE}, \ldots)$, rain intensity, number of lightning strikes inside the cell, velocity, and a few other more minor pieces of information.

The forecast horizon of $30 \mathrm{~min}$ was chosen as a compromise between the need to have enough time to manage the alarm and for a reliable forecast. The processing and SMS transmission takes only a few minutes to manage even a hundred or more targets and contemporary SMS messages.

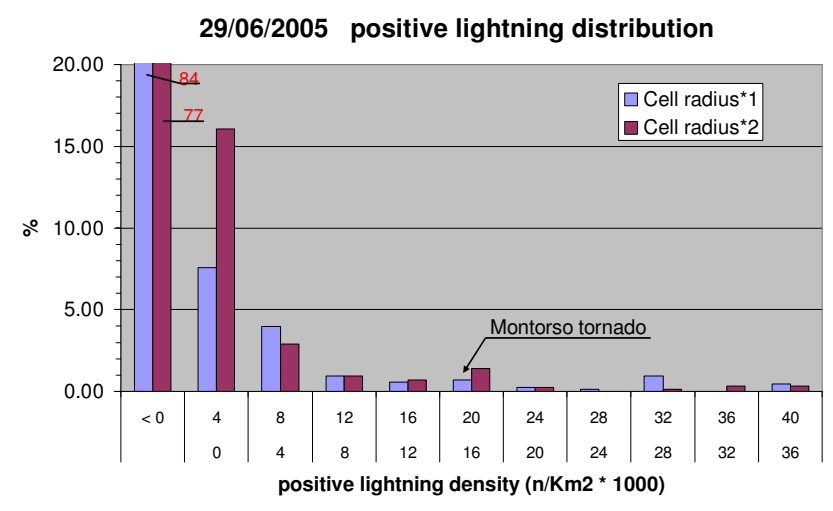

Fig. 13. Frequency distribution of positive lightning density inside a thunderstorm cell for all the cells tracked on 29 June 2005. The blue bars are related to lightning fallen inside an area with the same cell radius; the red bars are related to a double radius. The intervals on the $\mathrm{x}$-axis represent the values of positive lightning density. The Montorso tornado had a density between 16 and 20 .

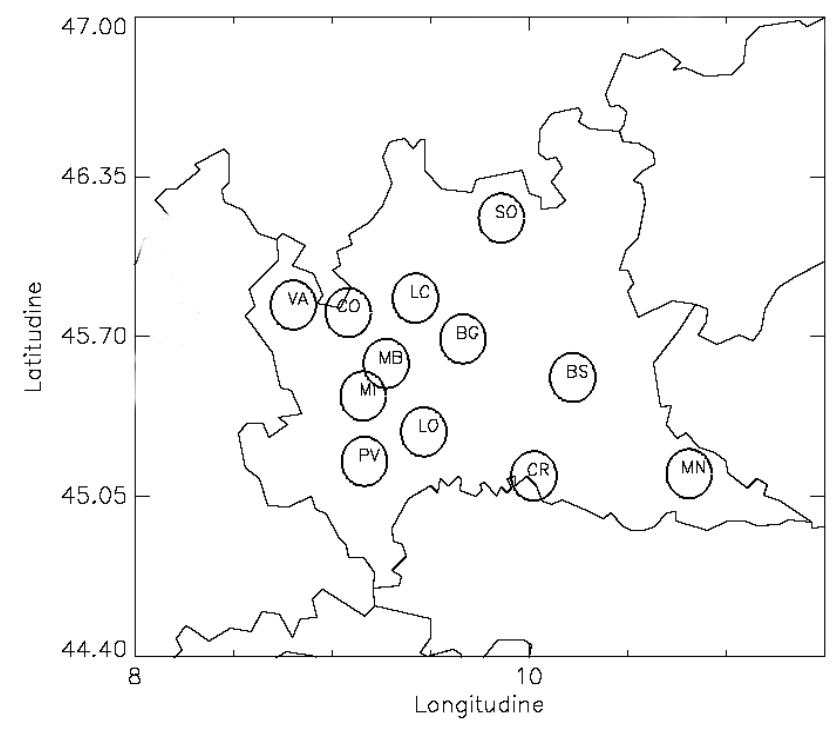

Fig. 14. Target areas (10 km radius) around 12 towns in Lombardy, used for the test campaign in the warm season of 2007.

\section{Assessment of the forecast}

How is it possible to judge whether or not a tracking algorithm is "good"? During the passage of a cold front, there are different scales of motions involved: thunderstorm cells have their own evolution, but they also move embedded in ensembles and interact among themselves. So when we look at two subsequent radar frames, it is not easy to identify the cell at the time $(t+d t)$ as the same cell that existed at the time $(t)$. This task is challenging for an automatic algorithm just as for human eyes looking at animated radar images. Nevertheless, for a warning system the most important thing is the final result; for this reason, in the context of the statement: 
Table 1. Verification scores for observed intensity $\geq 40 \mathrm{~mm} / \mathrm{h}$ and a target radius of $10 \mathrm{~km}$.

\begin{tabular}{lrrrrrrrrrrr}
\hline & \multicolumn{3}{c}{ Rain rate $\geq 40 \mathrm{~mm} / \mathrm{h}$} & \multicolumn{4}{c}{$30^{\prime}$ trajectory forecast } & \multicolumn{3}{c}{$30^{\prime}$ Persistence forecast } \\
town & alarms obs. & alarms forec. & POD & FAR & CSI & HSS & POD & FAR & CSI & HSS \\
\hline Bergamo & 142 & 137 & 0.55 & 0.43 & 0.39 & 0.55 & 0.53 & 0.47 & 0.36 & 0.52 \\
Brescia & 60 & 65 & 0.47 & 0.57 & 0.29 & 0.45 & 0.48 & 0.52 & 0.32 & 0.48 \\
Como & 145 & 137 & 0.52 & 0.45 & 0.36 & 0.53 & 0.52 & 0.48 & 0.36 & 0.52 \\
Cremona & 53 & 40 & 0.40 & 0.48 & 0.29 & 0.45 & 0.42 & 0.58 & 0.26 & 0.41 \\
Lecco & 162 & 152 & 0.44 & 0.53 & 0.29 & 0.45 & 0.44 & 0.56 & 0.29 & 0.44 \\
Lodi & 34 & 42 & 0.53 & 0.57 & 0.31 & 0.47 & 0.41 & 0.59 & 0.26 & 0.41 \\
Mantova & 39 & 29 & 0.36 & 0.52 & 0.26 & 0.41 & 0.38 & 0.62 & 0.24 & 0.38 \\
Milano & 83 & 68 & 0.46 & 0.44 & 0.34 & 0.50 & 0.42 & 0.58 & 0.27 & 0.42 \\
Monza & 126 & 124 & 0.54 & 0.45 & 0.37 & 0.54 & 0.50 & 0.50 & 0.33 & 0.49 \\
Pavia & 57 & 58 & 0.61 & 0.40 & 0.44 & 0.61 & 0.58 & 0.42 & 0.41 & 0.58 \\
Sondrio & 27 & 34 & 0.44 & 0.65 & 0.24 & 0.39 & 0.26 & 0.74 & 0.15 & 0.26 \\
Varese & 97 & 100 & 0.43 & 0.58 & 0.27 & 0.42 & 0.40 & 0.60 & 0.25 & 0.40 \\
\hline
\end{tabular}

"the best tracking algorithm is the one giving the best forecast", we have designed and tested a verification scheme in order to assess the skill of the algorithm and its set of parameters at forecasting the cell positions with half an hour lead time.

During the test campaign of 2007, twelve main towns in Lombardy were defined as target areas (specifically, circles with a radius of $10 \mathrm{~km}$ around every town). The test period ran from the 23 April to the 31 August 2007. The test included the verification of SMS arrival time to the staff of local Civil Defence users, the collection of subjective local observations about the thunderstorms' ground effects, and the verification of forecasted thunderstorm positions. The results of these last verifications are reported here.

We have checked only the forecasted and observed radar cells with maximum intensity greater than $40 \mathrm{~mm} / \mathrm{h}$ and that overlap for more than one pixel any of the 12 target areas. The presence/absence of a forecasted/observed cell in the area generates four possibilities for every $15 \mathrm{~min}$ time step. The time horizon of the forecast was $30 \mathrm{~min}$. The most used indexes to verify dichotomous forecasts have been computed. A discussion of these indexes used for the forecast of severe thunderstorms can be found in Kunz (Kunz, 2007).

Table 1 reports the results, divided by different town areas, obtained from the test season for a 30 min trajectory forecast and for a "persistence" forecast. The last was obtained from the same position of the observed cell in the target area, $30 \mathrm{~min}$ before the forecast time, and was compared with the observed cells at the forecast time. The first and second column after the town column in the table contain the total number of observed and forecasted thunderstorms, respectively. The other columns contain the values of four skill indexes, either for the trajectory or persistence forecast.

It is easy to see in Table 1 that the Heidke Skill Score (HSS), a generally used skill score that eliminates the effect of a purely random forecast, is a little higher for trajectory forecast than those obtained from persistence, except for the town of Brescia. The False Alarm Rate (FAR) also shows an improvement for the trajectory forecast, again except for Brescia, and similarly for the Critical Success Index (CSI). Probability of Detection (POD) is lower for trajectory than for persistence for Cremona and Mantova as well as Brescia.

It must be noted that the Brescia area is not well covered by radar due to some mountainous obstacles. This fact can lead to an under-estimate of the rain rate, sometimes causing it to vary abruptly over even short paths around the area, leading to failure in the detection of cells. In confirmation of this fact we can see, from the table, that the number of cells observed for Brescia are roughly half of those for Bergamo, about $40 \mathrm{~km}$ away.

\section{Conclusion}

A tracking algorithm, able to recognize heavy rainfall cells and to connect them between two radar time steps, has been developed and tested. The algorithm allows one to compute the velocity of the cells and to forecast their new positions after half an hour or more. This tracking method is also able to detect thunderstorm cells and follow their path by means of lightning position data alone. This is particularly useful in those regions where radar is not available.

Experiments of tracking cells, both from lightning data and from radar rainfall data, are carried out for northern Italy using a Meteo Swiss radar and the high resolution Lightning Location System of CESI-SIRF. The radar trajectories obtained were quite satisfactory for those thunderstorm systems moving regularly. Of course, locally developed and short lived cells cannot be tracked as well. Lightning trajectories 
seem to be more irregular and suggest their use primarily for larger scale systems.

The association of positive lightning density with a rain cell detected by radar, along its path, can be useful for investigating the relationship between this quantity and the occurrence of tornadoes, as some authors have suggested. A case of a documented tornado that occurred near the town of Vicenza, at the same time that an increase of CG positive lightning was observed, was discussed.

The tracking algorithm and its alert system was tested in the warm season of 2007, in collaboration with the Regione Lombardia Civil Defence, and a skill score scheme was applied in order to evaluate its performance. A $30 \mathrm{~min}$ forecast obtained by extrapolating radar cells positions has been compared with observed radar cells. Target areas of $10 \mathrm{~km}$ around the 12 main towns have been defined and the presence/absence of observed/forecasted thunderstorms have been counted. The resulting score indexes have been compared with those obtained from a persistence forecast. An improvement of scores are evident for the trajectory forecast system compared to persistence.

A longer forecast horizon of, for example, $1 \mathrm{~h}$, could be tested, although we expect that the scores might significantly decrease using the forecast system as currently implemented. Some improvements in forecast horizon could likely be achieved by tracking the whole mesoscale system instead of the single thunderstorm cell, but this task probably requires the availability of a coordinated radar network.

The algorithm can also likely be improved in the future using other radar products, such as parameters derived from the whole 3-D radar reflectivity, a 5 min time step, and geostationary satellite cloud top temperature in order to assess the dangerous ground effects of a thunderstorm, in addition to the heavy rain. Despite the short alert time permitted, a heavy thunderstorms alarm, received everywhere by cellphone, can be useful in those situations where rapid actions may be taken in order to place people in safety, as in, for example, recreational activity or traffic circulation. Other applications include the computer monitoring of a wide area by non-meteorologist staff in order to detect sites subjected to severe weather. This application may be useful for managing rapid rescue teams, such as for injured people or for overhead electrical lines.

Acknowledgements. This work has been financed by the Research Fund for the Italian Electrical System provided by the earlier Ministry of Production Activities (today the Ministry of Economic Development) and by the Regione Lombardia Civil Defence for the more recent development.

Authors are thankful to the staff of Meteo Swiss - Locarno Monti for their useful suggestions in using Monte Lema radar data and to Marina Bernardi of CESI S.p.A., Milano, Italy for her advices about lightning data. The authors are grateful to the two referees for their review work and valuable suggestions. Thanks also to C. P. Weaver for comments on the writing.
Edited by: A. Mugnai and C. Price

Reviewed by: two anonymous referees

\section{References}

Bernardi, M. and Ferrari, D.: Evaluation of the LLS efficiency effects on the ground flash density, using the Italian lightning detection system SIRF, J. Electrostatics, 60(2-4), 131-140, 2004.

Bertato, M., Giaiotti, D. B., Manzato, A., and Stel, F.: An interesting case of tornado in Friuli-Northeastern Italy, Atmos. Res., 67-68, 3-21, 2003.

Bertram, I. and Mayr, G. J.: Lightning in the eastern Alps 19931999, part I: Thunderstorm tracks, Nat. Hazards Earth Syst. Sci., 4, 501-511, 2004, http://www.nat-hazards-earth-syst-sci.net/4/501/2004/.

Bluestein, H. B. and MacGorman, D. R.: Evolution of cloud-toground lightning characteristics and storm structure in the spearman, Texas, tornadic supercells of 31 May 1990, Mon. Weather Rev., 126(6), 1451-1467, 1998.

Demetriades, N. W. S., Murphy, M. J., and Holle, R. L.: Long Range Lightning Nowcasting Applications for Meteorology, World Weather Research Programme Symposium on Nowcasting and Very Short Range Forecasting (WSN05), Toulouse, 5-9 September 2005.

Dixon, M. and Wiener, G.: TITAN: Thunderstorm Identification, Tracking, Analysis and Nowcasting - A Radar-based Methodology, J. Atmos. Oceanic Technol., 10, 785-795, 1993.

Hering, A. M., Morel, C., Galli, G., Sénési, S., Ambrosetti, P., and Boscacci M.: Nowcasting thunderstorms in the Alpine region using a radar based adaptive thresholding scheme, Proceedings of Third European Conference of Radar in Meteorology and Hydrology (ERAD), available on http://www.copernicus.org/, September 2004.

Hering, A. M., Sénési, S., Ambrosetti, P., and Bernard-Bouissières, I.: Nowcasting Thunderstorms in complex cases using radar data, World Weather Research Programme Symposium on Nowcasting and Very Short Range Forecasting (WSN05), Toulouse, 5-9 September 2005.

Johnson, J. T., MacKeen, P. L., Witt, A., Mitchell, E. D., Stumpf, G. J., Eilts, M. D., and Thomas, K. W.: The Storm Cell Identification and Tracking Algorithm: An Enhanced WSR-88D Algorithm, Weather Forecast., 13, 263-276, 1998.

Joss, J., Schadler, B., Galli, G., Cavalli, R., Boscacci, M., Held, E., Della Bruna, G., Kappenberger, G., Nespor, V., and Spiess, R.: Operational Use of Radar for Precipitation Measurements in Switzerland, NFP31: Projekt NOWRAD, Meteo Swiss, Locarno, 108 pp., 1997.

Kunz, M.: The skill of convective parameters and indices to predict isolated and severe thunderstorms, Nat. Hazards Earth Syst. Sci., 7, 327-342, 2007,

http://www.nat-hazards-earth-syst-sci.net/7/327/2007/.

MacGorman, D. R. and Rust, W. D.: The electrical nature of storm, Oxford University Press, 422 pp., 1998.

Poli, V., Alberoni, P. P., Paccagnella, T., and Cesari, D.: Preliminary results of the comparison of two advection methods, World Weather Research Programme Symposium on Nowcasting and Very Short Range Forecasting (WSN05), Toulouse, 5-9 September 2005 . 
Tessendorf, S. A., Miller, L. J., Wiens K. C., and Rutledge, S. A.: The 29 June 2000 Supercell Observed during STEPS. Part I: Kinematics and Microphysics, J. Atmos. Sci., 62(12), 41514177, 2005

Wilson, J. W., Crook, N. A., Mueller, C. K., Sun, J., and Dixon, M.: Nowcasting Thunderstorms: A Status Report, B. Am. Meteorol. Soc., 79(10), 2079-2099, 1998.
Wilson, J. W.: Precipitation nowcasting: Past, Present and Future, Sixth International Symposium on Hydrological Application of Weather Radar, Melbourne, Australia, 2-4 February 2004.

$\mathrm{Xu}, \mathrm{G}$. and Chandrasekhar, V.: Radar storm motion estimation and beyond: a spectral algorithm and Radar observation based dynamic model, World Weather Research Programme Symposium on Nowcasting and Very Short Range Forecasting (WSN05), Toulouse, 5-9 September 2005. 